\title{
High resolution X-ray diffraction studies of real structure of nearly perfect single crystals
}

\author{
KRISHAN LAL \\ National Physical Laboratory, New Delhi 110012, India
}

\begin{abstract}
Real structure together with composition and elemental purity of single crystals controls their properties. This paper reviews recent work carried out at the National Physical Laboratory on application of high resolution X-ray diffractometry, topography and diffuse $\mathrm{X}$-ray scattering for direct observation and characterization of real structure of single crystals of silicon, gallium arsenide, diamond and $\mathrm{LiNbO}_{3}$. A series of six multicrystal X-ray diffractometers have been designed, developed and fabricated indigenously. The most versatile of these systems is a five crystal X-ray diffractometer with state-of-the-art level resolution. These techniques and equipments have been applied in studying several interesting problems. Even in dislocation-free crystals of silicon, remarkable differences in the defect structure have been observed if the growth method was changed from float zone to Czochralski. Study of effect of externally applied electric fields and ion implantation on real structure of crystals has yielded interesting results. Images of 'filaments' which show nonhomogeneous distribution of electric current through semiconductors and insulators have been recorded for the first time in high resolution traverse topographs. Diffracted X-ray intensities could be modified by externally applied electric fields. It has been shown that implantation of $\mathrm{BF}_{2}^{+}$ions in silicon for producing shallow junctions does not produce homogeneous distribution of boron. The impurity is partially in clustered form. Biaxial stress introduced by thin depositions in substrate crystals are of considerable applied concern. The value and nature of stress have been determined in a number of systems. Typical results obtained on GaAs: multilayer metallizations are described. Also, degradation of perfection of substrates has been monitored. This work has shown that the stress is not homogeneously distributed and is quite anisotropic. A new high resolution X-ray diffraction technique has been developed for direct observation and study of forward diffracted X-ray beam and anomalous transmission of $\mathrm{X}$-rays through 'thin' diamond crystals of varying degrees of perfection.
\end{abstract}

Keywords. Single crystals; real structure; high resolution X-ray diffraction.

\section{Introduction}

Well characterized materials form the backbone of modern technological advances and industrial production based on the same. Also, for fundamental understanding of the behaviour of materials, it is necessary to have a correlation between their properties and the basic materials characteristics, namely composition, purity, crystallographic structure and crystal defects (NAS and NAE Report 1967; Laudise 1975; Lal 1988a). Continued $R$ and $D$ efforts lead to improvement in characterization techniques as well as in production of materials and in their applications in highly advanced areas. An excellent example is silicon crystals for microelectronic applications. Industrial demands are forcing growth of large size crystals and the miniaturization of devices demands homogeneities at micron and submicron levels. Therefore, characterization techniques have to be continuously upgraded to meet the ever increasing challenges.

For observation and characterization of crystal defects, a variety of techniques like etching, transmission electron microscopy, birefringence and high resolution X-ray 
diffractometry and topography are available. X-ray diffraction techniques have remarkable features. These are non-destructive in character and can be used to characterize large volumes of crystals regarding all types of defects. Further, changes in real structure caused by external influences such as heat treatment or incorporation of impurities by diffusion or implantation can be monitored. Sustained efforts at the National Physical Laboratory (NPL) have led to the development of a wide variety of high precision techniques employing high resolution X-ray diffraction. Also, a series of multicrystal X-ray diffractometers including a five-crystal X-ray diffractometer have been designed, developed and fabricated in the laboratory (Lal and Singh 1977, 1979; Lal and Verma 1984; Lal and Goswami, to be published). In this paper, we shall describe: basic principles of the techniques of high resolution X-ray diffraction; a five-crystal X-ray diffractometer; typical results of characterization of defects in as grown crystals; characterization of defects induced due to processing steps required in fabrication of microelectronic devices; microstructural effects generated by external electric fields in silicon single crystals; and direct observation of a forward diffracted beam in thin diamond crystals of varying degrees of perfection.

\section{Theoretical aspects}

Results of X-ray diffraction experiments are explained on the basis of either of the two prominent theories, namely the kinematical theory and the dynamical theory (Darwin 1914; Zachariasen 1945; James 1950; Pinsker 1978). For diffraction from nearly perfect crystals, the dynamical theory is widely used (Darwin 1914; Ewald 1916, 1917; Zachariasen 1945; von Laue 1960; James 1963; Batterman and Cole 1964; Kato 1974; Pinsker 1978). In this theory, drastic simplifying assumptions of the kinematical theory regarding absence of rescattering of $X$-rays and constancy of intensity of the exploring beam throughout the irradiated volume are not the constraining factors. Each atom inside the crystal is assumed to receive scattered waves from all the atoms in the crystal, in addition to the exploring beam whose intensity has been weakened by photoelectric absorption and scattering as it traverses the diffracting crystal. Maxwell's equations of electromagnetic radiation are solved inside the crystal. The periodic arrangement of constituent atoms and ions is taken into account by assuming the dielectric constant to have the periodicity of the lattice. Appropriate boundary conditions are imposed for reflection geometry (Bragg case) and transmission geometry (Laue case). If the exploring beam is nearly parallel and monochromated the plane wave dynamical theory can be employed. According to this theory, the diffraction curves for Bragg case can be obtained from the following relation (Batterman and Cole 1964):

$$
\frac{I_{H}}{I_{0}}=|b|\left|\eta \pm\left(\eta^{2}-1\right)^{1 / 2}\right|^{2}
$$

Here $b$ is the asymmetric factor, $=-1$ for symmetrical Bragg case, and $\eta$ is a function of angular separation $\Delta \theta$ from the diffraction peak, structure factor $F_{H}$ of the reflection under consideration, and polarization of the beam, and is given by

$$
\eta=\left[b \Delta \theta \sin 2 \theta+(1 / 2) \Gamma F_{0}(1-b)\right] / \Gamma|P||b|^{1 / 2}\left[F_{H} F_{H}\right]^{1 / 2}
$$


Similarly, diffraction curves for the Laue case can be obtained from the following expression (Batterman and Cole 1964):

$$
\frac{I_{H \omega}}{I_{0}}=\frac{1}{4}\left[\frac{1}{\left(1+\eta^{\prime 2}\right)^{1 / 2}}\right] \exp \left[-\frac{\mu_{0} t_{0}}{\gamma_{0}}\left\{1 \mp \frac{|P| \varepsilon}{\left(1+\eta^{\prime 2}\right)^{1 / 2}}\right\}\right] .
$$

Here $\omega$ refers to the $\alpha$ and $\beta$ branches of the dispersion surface; the upper sign (negative) refers to the $\alpha$ and the lower sign to the $\beta$ branch.

The half width of these curves can be expressed in terms of the structure factor of the reflection, the wavelength of the exploring beam, the Bragg angle and the volume of the unit cell. For the Bragg geometry the half width is given by

$$
\Delta \theta=\frac{2 r_{e} \lambda^{2}}{\pi V_{c} \sin 2 \theta_{B}} F_{H}^{\prime}|P|
$$

For divergent exploring X-ray beams, spherical wave dynamical theory has been developed (Kato 1974; Pinsker 1978).

All real crystals are not absolutely perfect. Atomic arrangement in these crystals can be disturbed in local regions. Kato and others have developed a statistical dynamical theory by describing diffraction of X-rays from real crystals (Kato 1980; Schneider et al 1992).

Two of the most outstanding results of the dynamical theory are the Pendellosung phenomena (Batterman and Cole 1964; Kato 1974; Tanner 1976; Pinsker 1978) and the anomalous transmission of X-rays or the Borrmann effect (Borrmann 1950; Batterman and Cole 1964; Pinsker 1978). The former demands swapping of energy between the forward diffracted beam and the diffracted beam as a function of depth inside the crystal. The latter shows loss in absorption as the crystal is oriented for diffraction of X-rays. To observe Borrmann effect, the crystal needs to be nearly perfect and quite thick, defined by $\mu t \geqslant 10$. Here $\mu$ is the linear absorption coefficient of the crystal for the exploring $X$-ray beam and $t$ is its thickness. Recent experiments in our laboratory have shown that if a high quality $\mathrm{X}$-ray beam is utilized, forward diffracted beam and the enhanced transmission of X-rays can be observed even when $\mu t$ is $<1$ and the crystals are not so perfect (Lal et al 1992, 1993).

Even dislocation-free crystals are not absolutely perfect. These contain point defects which are unavoidable thermodynamically at any finite temperature. Generally, crystals contain point defects far in excess of the thermodynamically expected concentrations. In addition, there are point defect clusters. It is not possible to use X-ray diffractometry and $X$-ray diffraction topography to characterize these. In such a situation, study of $X$-ray scattering close to $X$-ray diffraction maxima is valuable. $X$-ray scattering from the reciprocal space around reciprocal lattice points is very sensitive to the presence of point defects and their aggregates. In absolutely perfect crystals, the scattering from this region can be calculated on the basis of dynamical theory, as mentioned above. It is expected to be negligible or close to zero. However, even a small disturbance produced by point defect clusters in the lattice will produce finite scattering. The fractional change in the scattered intensity due to defects is very large in angular regions around the peak compared to that at the peak of diffraction maxima. This type of scattering has come to be known as diffuse X-ray scattering (DXS) (Wooster 
1962; Krivoglaz 1969; Dederichs 1973; Patel 1975; lida and Kohra 1979; Lal et al 1979; Ehrhart et al 1982; Zaumseil et al 1987; Lal 1989, 1991a). It is to be kept in view that diffuse scattering is also produced by elastic thermal waves or phonons (Wooster 1962). Also, whenever a crystal is irradiated with X-rays, inelastic scattering is also produced (Compton and Allison 1935). It is possible to differentiate between the contributions of different sources of diffuse scattering and isolate contribution of point defect clusters. Indeed, a detailed investigation at NPL had shown that at near the room temperature, the scattering from elastic thermal vibrations of the lattice is negligible, in comparison to that from the defects (Lal 1982, 1989).

It is well known that the intensity of diffracted beam from a set of lattice planes of an absolutely perfect crystal defined by reciprocal lattice factor $\mathbf{R}^{*}$ is given by

$$
I\left(\mathbf{R}^{*}\right) \propto\left|\sum_{m} f_{m}\left(\mathbf{R}^{*}\right) \exp 2 \pi i\left(\mathbf{R}^{*} \cdot \mathbf{r}_{m}\right)\right|^{2},
$$

where $f_{m}$ is the atomic scattering factor for the atom $m$ whose position is defined by vector $\mathbf{r}_{m}$ which defines a perfectly periodic lattice so that

$$
\mathbf{r}=p \mathbf{a}+q \mathbf{b}+\mathbf{r c},
$$

where $(p, q, r)$ are integers and $(\mathbf{a}, \mathbf{b}, \mathbf{c})$ are the basic translation vectors of the crystal lattice.

The presence of point defects and their clusters disturbs the positions of atoms on the perfect periodic network. The deviation from the ideal position can be denoted by a small vector $\mathbf{u}_{m}$ for an atom defined by $\mathbf{r}_{m}$. In general, for a crystal containing defects, the scattered intensity will be given by

$$
I\left(\mathbf{R}^{*}+\mathbf{K}^{*}\right)=\left|\sum_{m} f_{m}\left(\mathbf{R}^{*}\right) \exp 2 \pi i \mathbf{H}^{*} \cdot\left(\mathbf{r}_{m}+\mathbf{u}_{m}\right)+\sum_{D} f_{D}\left(\mathbf{R}^{*}\right) \exp 2 \pi i \mathbf{H}^{*} \cdot \mathbf{r}_{D}\right|^{2},
$$

where $f_{D}$ is the scattering factor of the species occupying defect sites and $\mathbf{H}^{*}=\mathbf{R}^{*} \pm$ $\mathbf{K}^{*}, \mathbf{K}^{*}$ being the vector which joins the elemental volume producing scattering to the reciprocal lattice point defined by $\mathbf{R}^{*}$. This general equation cannot be rigorously solved. Model calculations are, however, possible. In a specific case of clusters of radius $R_{\mathrm{cl}}$ the DXS intensity at a point away from the reciprocal lattice point defined by $K^{*}$ based on a phenomenological model is given by (Dederichs 1973):

$$
\begin{aligned}
& I\left(\mathbf{R}^{*}+\mathbf{K}^{*}\right)=C_{c l}\left|F_{P, T}\right|^{2} {\left[-\frac{4 \pi A_{c l}}{V_{c}} \frac{\mathbf{R}^{*} \cdot \mathbf{K}^{*}}{\mathbf{K}^{* 2}} \frac{\sin \mathbf{K}^{*} \cdot R_{c l}}{\mathbf{K}^{*} \cdot R_{c l}}-\right.} \\
&\left.\frac{4 \pi R_{c l}^{3}}{V_{c}}\left\{\frac{\sin \mathbf{K}^{*} \cdot R_{c l}-\mathbf{K}^{*} R_{c l} \cos \mathbf{K}^{*} \cdot R_{c l}}{\left(\mathbf{K}^{*} \cdot R_{c l}\right)^{3}}\right\}\right]^{2},
\end{aligned}
$$

where $C_{c l}$ is the cluster density; $F_{P, T}$ is the structure factor corrected for polarization and temperature, $A_{c l}=R_{c l}^{2} / \mathbf{R}^{*}$ and $V_{c}$ is the volume of the unit cell. This relation quantitatively gives the variation of the DXS intensity as a function of $\mathrm{K}^{*}$. At small values of $\mathbf{K}^{*}$ the first term dominates and the intensity varies as $\mathbf{K}^{*-2}$ as in the case of Huang scattering from isolated point defects. At large values of $\mathbf{K}^{*}, I\left(\mathbf{R}^{*}+\mathbf{K}^{*}\right)$ varies as $\mathrm{K}^{*-4}$ (Stokes-Wilson scattering) and the second term in (6) is dominant. 
By fitting the experimental data on theoretical curves the cluster size can be determined. Other approaches for analysis of scattering have also been developed (Ehrhart et al 1982).

\section{Experimental conditions}

Ideally, one would require a perfectly parallel and monochromated X-ray beam to perform high resolution X-ray diffraction experiments. However, in actual practice, it is not easy to get an X-ray beam satisfying these conditions. There is always a finite wavelength and direction spread. If these spreads can be reduced below the intrinsic half widths of diffraction curves to be recorded, quality of the beams can be considered to be satisfactory. By using long collimators combined with fine slits and small sized sources, it is possible to reduce the divergence to less than a minute of arc or so, and at the same time obtain exploring beams with reasonably good intensities. If such well collimated X-ray beams are diffracted from nearly perfect crystals, the wavelength purity and divergence of the diffracted beam can be reduced to very low levels. By a combination of multiple diffractions, one can obtain high quality X-ray beams with which diffraction curves from nearly perfect crystals can be recorded without any appreciable broadening. In the following, we shall describe a five crystal X-ray diffraction system which employs a combination of three plane silicon crystal monochromators - collimators which give nearly parallel and monochromated X-ray beam (Lal and Goswami, to be published):

\section{A five-crystal $X$-ray diffractometer}

Figure 1 shows a schematic line diagram of a five-crystal X-ray diffractometer designed, developed and fabricated at NPL (Lal and Goswami, to be published). A photograph of this diffractometer is shown in figure 2 . The first three crystals of this diffractometer

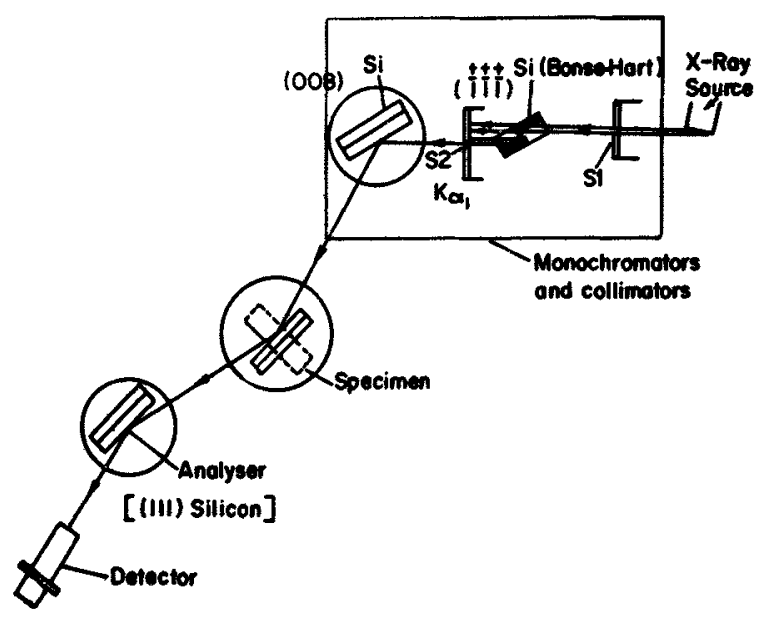

Figure 1. A schematic line diagram of the five-crystal $X$-ray diffractometer designed and developed at the National Physical Laboratory, New Delhi. 


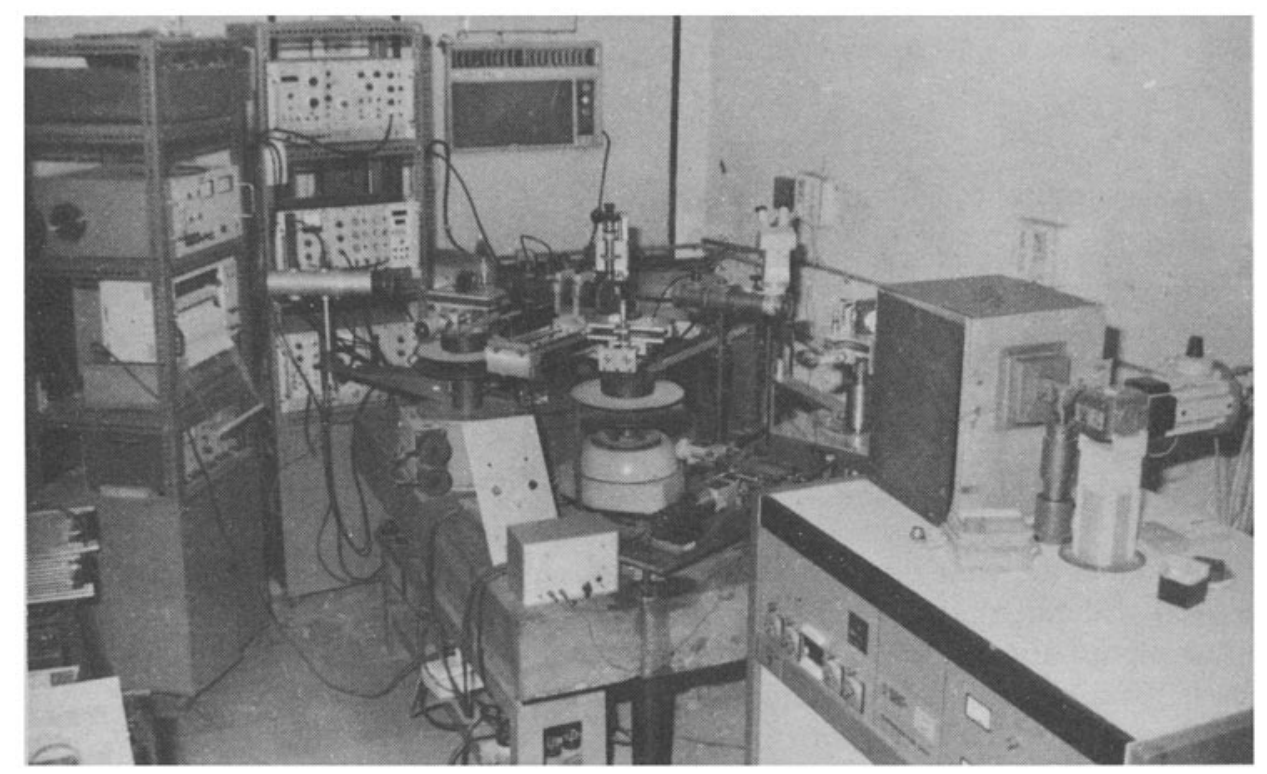

Figure 2. A photograph of the five-crystal X-ray diffractometer designed, developed and fabricated at the National Physical Laboratory, New Delhi.

are monochromators and collimators. The specimen is generally at the fourth crystal position and the fifth crystal can be used as an analyser. If necessary, the specimen can be shifted to the fifth crystal position or even at the first crystal position. In an extreme case, all the monochromators can be removed and the diffractometer can function like a Lang camera. X-ray beam from a fine focus X-ray source (Phillips; sealed tube $2 \mathrm{~kW}$ for molybdenum) is first collimated with the help of a $300 \mathrm{~mm}$ long collimator fitted with two sets of slits whose widths can be varied continuously. The plane of diffraction is horizontal and the width of the vertical slit is most crucial: It is adjusted to match the source size after foreshortening (about $0.4 \mathrm{~mm}$ for fine focus source). The width of the horizontal slit can be adjusted to get the desired height of the exploring X-ray beam emerging from the collimator. The divergence of the collimated beam is a few minutes of arc in the plane of diffraction. It is diffracted from a set of two plane silicon monochromator crystals of Bonse-Hart type set in $(+,-)$ geometry (Bonse and Hart 1965). Their diffracting surfaces are parallel to (111) planes. At this stage the $K \alpha_{1}$ component of the characteristic radiation $K \alpha$ is isolated. This beam is further diffracted from another plane silicon monochromator whose diffracted surface is along (100) planes. It is oriented for diffraction from (800) planes in $(+,-,+)$ geometry. This arrangement enables us to isolate a small fraction of the wavelength and the direction spread of the $K \alpha_{1}$ beam falling on the third crystal. This beam is used as the exploring beam for the specimen crystal which occupies the fourth crystal stage. The beam diffracted from the specimen falls on the analyser crystal to monitor small variations in lattice parameter in the specimen crystal from one region to another or due to externally applied disturbances like electric field. In study of electric field induced changes in silicon and other crystals, the analyser crystal has proven to be of considerable value (Lal and Thoma 1983; Lal and Goswami 1987; Lal 1988b). 
The third, fourth and fifth crystal stages of the diffractometer have provision for the following motions/translations:

(i) rotation around the vertical axis to change glancing angle $\theta$. This motion is provided with the help of a versatile turntable developed in our laboratory. Minimum rotations of about $0.1 \mathrm{sec}$ can be imparted to the crystal under investigation.

(ii) Tilt of the specimen across the X-ray beam so that the diffracting planes are along a vertical plane and the axis of the main turntable lies on the same. A special device has been developed in our laboratory which enables us to provide minimum tilt of 10 arcsec.

(iii) Azimuthal rotations of the specimens so that the desired plane of reciprocal space is brought in coincidence with the plane of diffraction.

(iv) The linear motion of the specimen in the plane of diffraction across the X-ray beam. This motion is provided with the help of a traversing device which produces homogeneous and uniform motions. The position of the crystal can be determined with a resolution of $0.01 \mathrm{~mm}$.

A combination of these motions enables one to orient a given crystal either in the Bragg or the Laue geometry. High resolution X-ray diffraction curves and topographs can be recorded. Distribution of diffusely scattered radiation near the reciprocal lattice points can be investigated. Bending of single crystal wafers can be measured with a high degree of sensitivity. Lattice mismatch between epitaxial layers and their substrates can be conveniently measured. If required, composition of epitaxial films can be determined from the observed lattice mismatch. Lattice parameter of the substrate of a single crystal can be determined with a high level of sensitivity $(1-10 \mathrm{ppm})$. Also, this diffractometer can be used for measurement of crystallographic orientation of visible surfaces of single crystal wafers and discs. This system can also be used for measurement of crystallographic orientation of straight edges of device quality wafers.

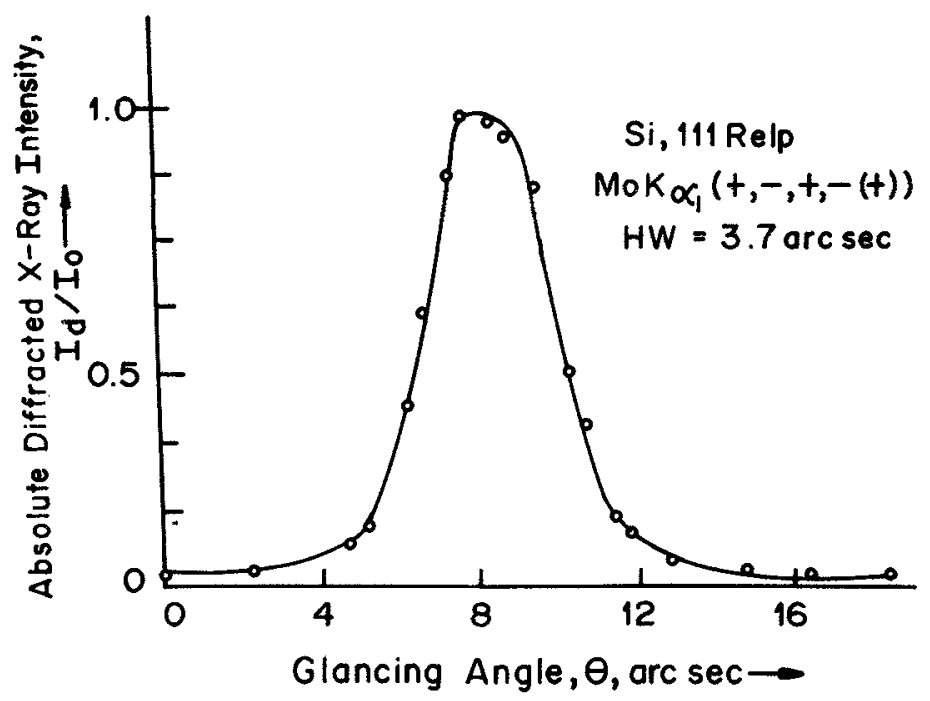

Figure 3. A typical diffraction curve of a nearly perfect silicon single crystal recorded on the five crystal $\mathrm{X}$-ray diffractometer. (111) diffracting planes, $\mathrm{Mo} \mathrm{K} \alpha_{1}$ radiation: $(+,-,+,-,+)$ configuration and symmetrical Bragg geometry were employed. 


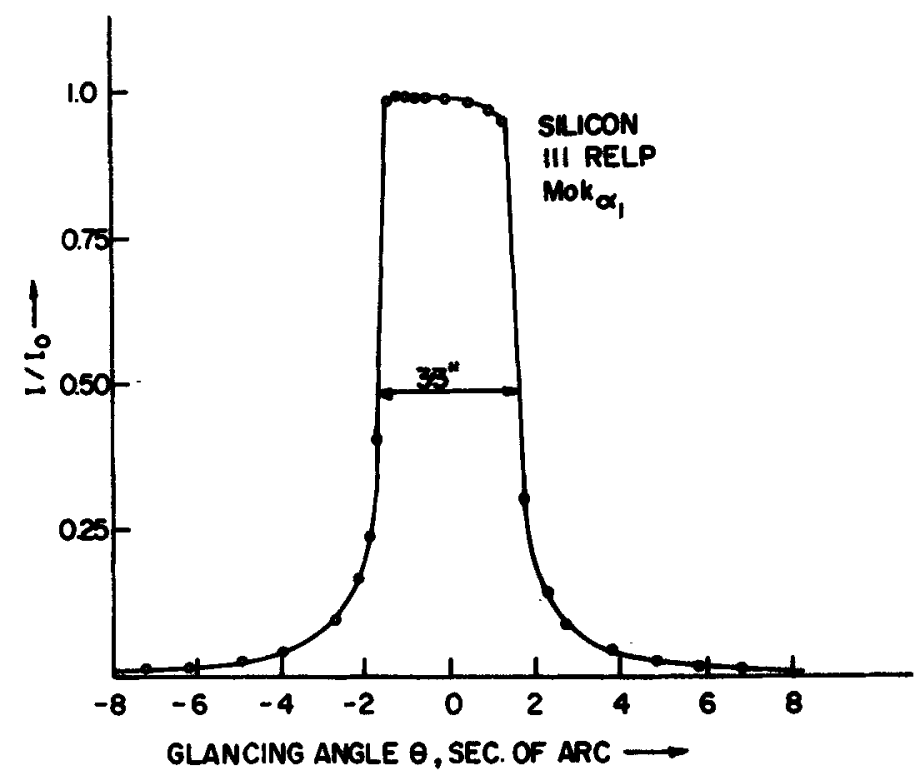

Figure 4. A theoretical diffraction for (111) diffracting planes of an ideally perfect silicon single crystal obtained on the basis of plane wave dynamical theory of $\mathrm{X}$-ray diffraction.

Both these techniques for determination of orientation have been developed in our laboratory and have unprecedented resolution (Lal and Goswami 1988; Lal et al 1990).

To demonstrate the quality of experiments that can be performed with the five crystal system, we show in figure 3 a typical diffraction curve of a device quality silicon single crystal. This is an as-recorded curve without any correction for experimental uncertainties. For the sake of comparison, in figure 4 we show a diffraction curve obtained theoretically for the same reflection and computed for experimental conditions of figure 3. Plane wave dynamical theory has been used to obtain the same (Batterman and Cole 1964). It is seen that both the experimental and theoretical curves show nearly $100 \%$ reflectivity at the diffraction peak position. The half width of the experimental curve ( 3.7 arcsec) is very close to that of the theoretical curve $(3.3 \mathrm{arcsec})$. The difference is about one-tenth of the theoretical half width. This remarkable feature gives the diffractometer a high degree of sensitivity. There are differences in the wings of the diffraction curve which are due to the presence of point defect clusters in silicon single crystals as shown elsewhere (Lal 1989). Another remarkable feature is that if diffraction from another set of planes such as $(\mathbf{4 0 0})$ planes is investigated the diffraction curve width is close to that expected for this reflection. The dispersion correction due to lattice mismatch between monochromators and specimen is negligible.

5. Some typical examples of characterization of crystal defects in single crystals of technological importance

We shall describe a few representative examples to illustrate the role of high resolution $\mathrm{X}$-ray diffraction techniques in observation and characterization of lattice imperfections. 
These will cover: (i) as-grown crystals; (ii) crystals subjected to processing for device fabrication; and (iii) crystals subjected to external electric fields.

\subsection{Crystal defects in as-grown crystals}

The following types of main defects can be present in a crystal: grain boundaries; subgrain or low angle boundaries; dislocations and point defects and their clusters. A specimen with grain boundaries is not a single crystal and generally not useful for device fabrication. It is fairly straightforward to observe and characterize grain boundaries by etching or by standard X-ray Laue technique.

A subgrain or small angle boundary separates two regions of a single crystal whose crystallographic orientations differ by a few minutes of arc or more. Standard X-ray diffraction topography method can conveniently reveal the presence of low angle boundaries and can characterize the same. The diffraction curves of such crystals split up into several peaks, each originating from a subgrain in the crystal (Lal 1982). From the angular separation between different peaks one can find out the angle between various subgrains. The diffraction curves extend over a range of many minutes of arc. It is possible to orient the crystal on any peak in the diffraction curve and record traverse topographs. This gives contours of the subgrains and will also reveal presence of other defects like dislocations in individual subgrains. Figure 5 shows a typical diffraction curve of a natural diamond crystal showing the presence of low angle boundaries (Lal et al 1990a). With the high resolution X-ray diffraction techniques

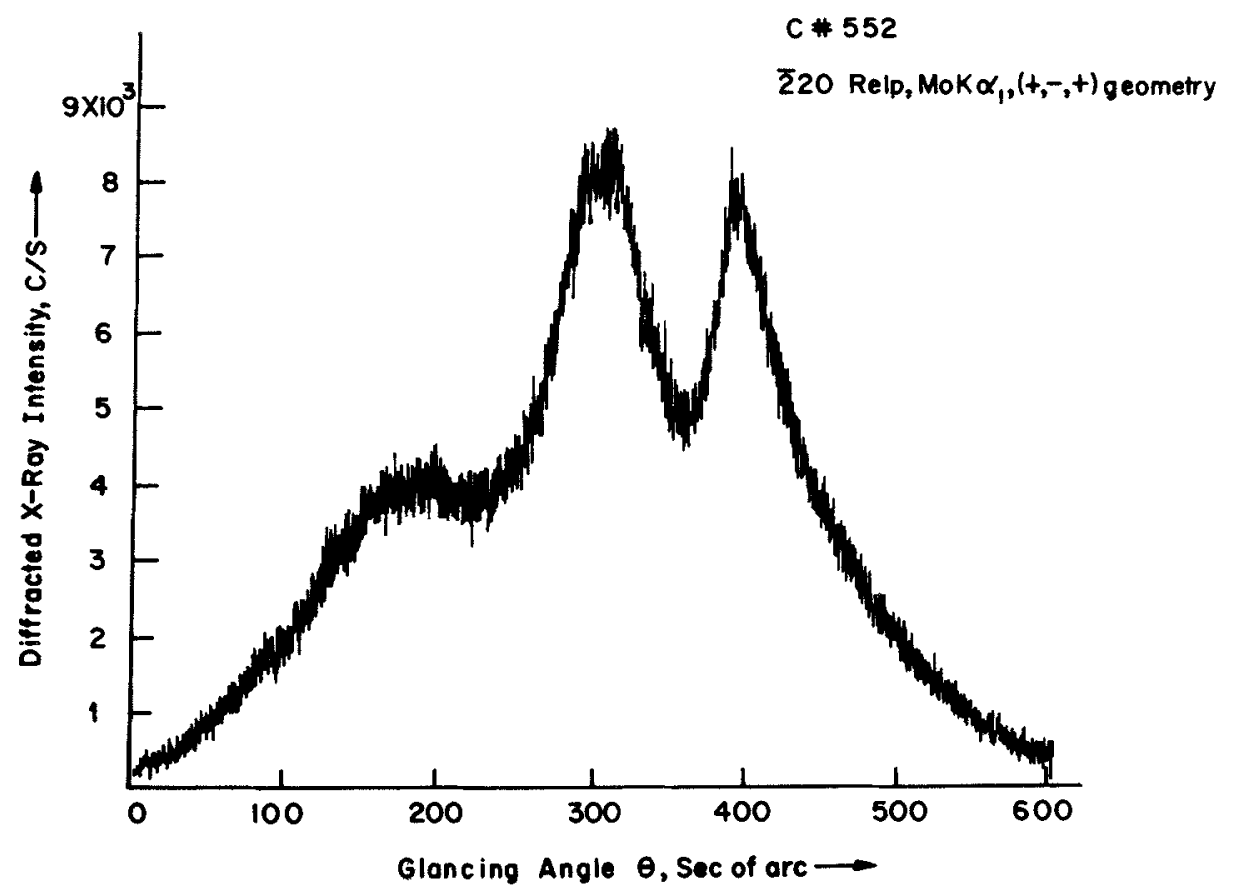

Figure 5. A diffraction curve of a (111) natural diamond platelet recorded by using a Mo K $\alpha_{1}$ exploring beam, $(\overline{2} 20)$ diffracting planes and symmetrical Laue geometry. Three peaks due to three subgrains in the irradiated volume are observed. 
described here, it has been possible to detect low angle boundaries with angles of less than an arcmin also.

Dislocations in crystals having densities below $10^{5}-10^{6} \mathrm{~cm}^{-2}$ can be directly observed and characterized by using standard X-ray diffraction topography methods. The limitation on the dislocation densities which can be resolved is primarily due to the limited spatial resolution of the photographic films or plates used for recording the topographs.

Diffraction curves of nearly perfect crystals which are free of boundaries and have low dislocation densities, when recorded by using the topographic system, consist of well resolved peaks due to $K \alpha_{1}$ and $K \alpha_{2}$ components of the $K \alpha$ doublet. Half widths of these maxima depend on the divergence and the wavelength spreads of the exploring X-ray beam. Their values are typically 40-60 arcsec (Lal 1982).

The multicrystal X-ray diffractometers have very high sensitivity to detect strain in nearly perfect crystals due to high quality of the exploring $X$-ray beam. This is illustrated in figure 6 which shows a traverse topograph of a semi-insulating GaAs single crystal grown by the horizontal Bridgman method (Lal et al 1990c). This was recorded with only the first two monochromators of the diffractometer (figure 1). Images of three dislocations are seen clearly. It may be mentioned that the half width of the diffraction curves of these crystals was about 15 arcsec with silicon crystals as monochromators. The strong contrast around dislocation images and its long range in this topograph illustrates the point.

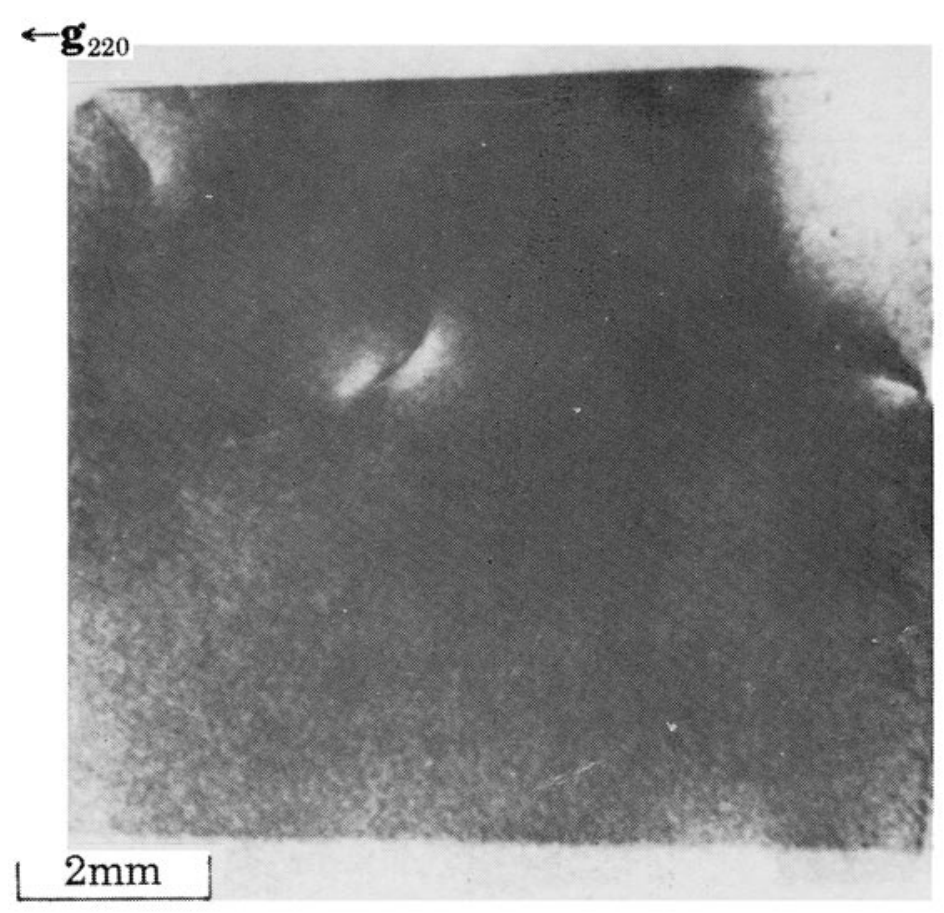

Figure 6. A high resolution X-ray diffraction topograph of a semi-insulating gallium arsenide crystal, recorded with $\mathrm{Mo} K \alpha_{1}$ exploring beam, (220) diffracting planes and $(+,-,+)$ setting of the diffractometer in symmetrical Laue geometry. 


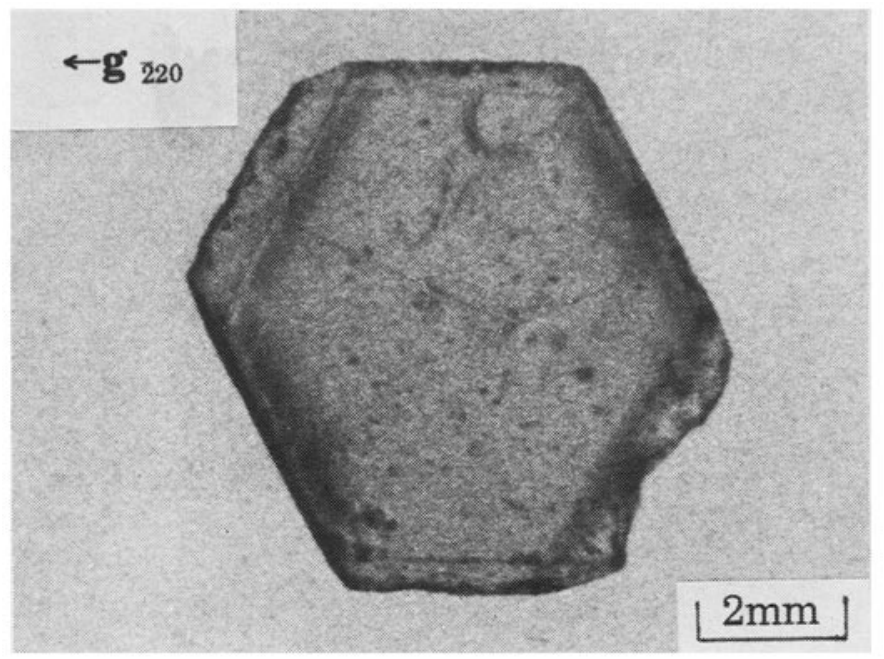

Figure 7. A high resolution traverse topograph of a (111) natural diamond crystal of type I variety recorded with $(\overline{2} 20)$ diffracting planes, symmetrical Laue geometry, $(+,-,+)$ setting of the diffractometer and $\mathrm{Mo} \mathrm{K} \alpha_{1}$ exploring beam.

We have investigated perfection of natural diamond crystals by high resolution $\mathrm{X}$-ray diffractometry and topography. These are from the famous collection of late Prof. C V Raman (courtesy, Raman Research Institute, Bangalore). The diamond crystals have varying degrees of perfection. Some of the crystals are quite imperfect with diffraction curve widths of 300 arcsec, i.e. about 300 times the theoretically expected half width for these crystals. Some of the crystals are quite perfect (Lal et al $1990 \mathrm{~b}, 1992)$ and gave very narrow diffraction curves with half width of about 10 arcsec or even less. Topographic examination of these crystals is also consistent with this result. Figure 7 shows a typical topograph of one such crystal (Lal et al 1992). It can be seen that the intensity is fairly uniform and no line defect is visible. For comparison figure 8 shows a traverse topograph of a device quality dislocation-free silicon single crystal. This specimen is also free of line defects (Lal 1991b). The observed texture is due.to the high resolution of the technique.

We shall consider a typical result of characterization of point defect clusters in dislocation-free silicon single crystals grown by the two most widely used techniques, namely the Czochralski and the float zone method (Lal and Bhagavannarayana 1989). A multicrystal X-ray diffractometer developed in the author's laboratory which employs a set of three plane monochromators oriented in $(+,-,-)$ configuration was used. Diffraction curves of all the specimens were very sharp, with half widths in the range 4-10 arcsec, close to the theoretically expected values for an ideal crystal. DXS intensity distribution around such diffraction maxima is measured close to the reciprocal lattice point at small angular intervals of a few seconds each. Figures 9(a) and (b) show typical DXS intensity versus $1 / \mathbf{K}^{* 2}$ plots for the Czochralski grown and the float zone grown crystals respectively. These results have been analysed on the basis of a model for dislocation loops (Dederichs 1973). This analysis shows that the defect clusters responsible for DXS from Czochralski grown crystals are of interstitial type. Further, the point defects have clustered in the form of interstitial dislocation loops. 


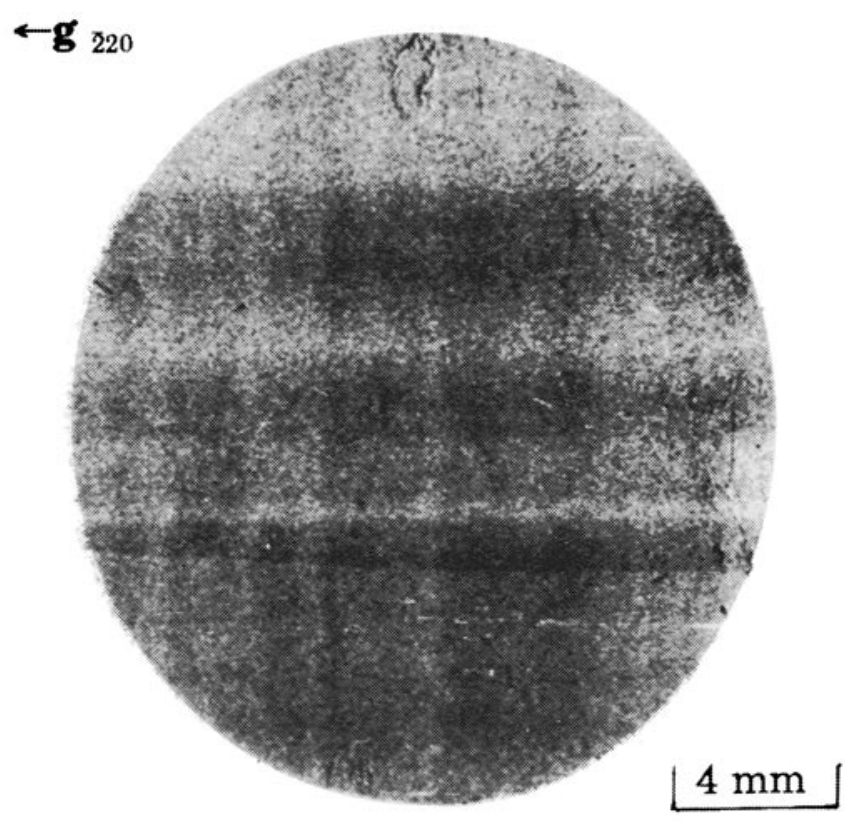

Figure 8. A typical high resolution $X$-ray diffraction topograph of a (111) silicon single crystal. Experimental conditions as in figure 7.

Two types of clusters with sizes $5.5 \times 10^{-5} \mathrm{~cm}$ and $3 \times 10^{-4} \mathrm{~cm}$ were observed in these crystals. Oxygen seems to be responsible for the same. The DXS intensity distribution from silicon single crystals grown by the float zone (FZ) method is remarkably different from that from the Czochralski grown crystal (figure $9 \mathrm{~b}$ ). In this case, platelet-shaped clusters of vacancies were the main defects. Typical sizes of these clusters are: $2 \times 10^{-5} \mathrm{~cm}$ and $2.6 \times 10^{-4} \mathrm{~cm}$.

Diffuse X-ray scattering measurements have been extensively used to study changes in clusters produced by heat treatments (Patel 1973; Lal and Singh 1980). Also, defects generated by ion implantation and other steps used to fabricate devices on crystals can be characterized (Lal and Bhagavannarayana 1991).

\subsection{Characterization of process induced defects in crystals}

Processing of single crystals for device fabrication generally does not produce significant changes in the composition and the 'ideal' structure or crystallographic phase. Main effect is on purity and defect structure. Here we shall consider examples which illustrate the role of $\mathrm{X}$-ray diffraction techniques for characterization of changes in real structure of device quality crystals as affected by important processing steps.

5.2a Investigation of biaxial stress due to thin metal deposits in gallium arsenide substrates: Metallization to produce ohmic contacts which are non-alloyed and stable at high temperatures is an important part of the technology needed for fabrication 

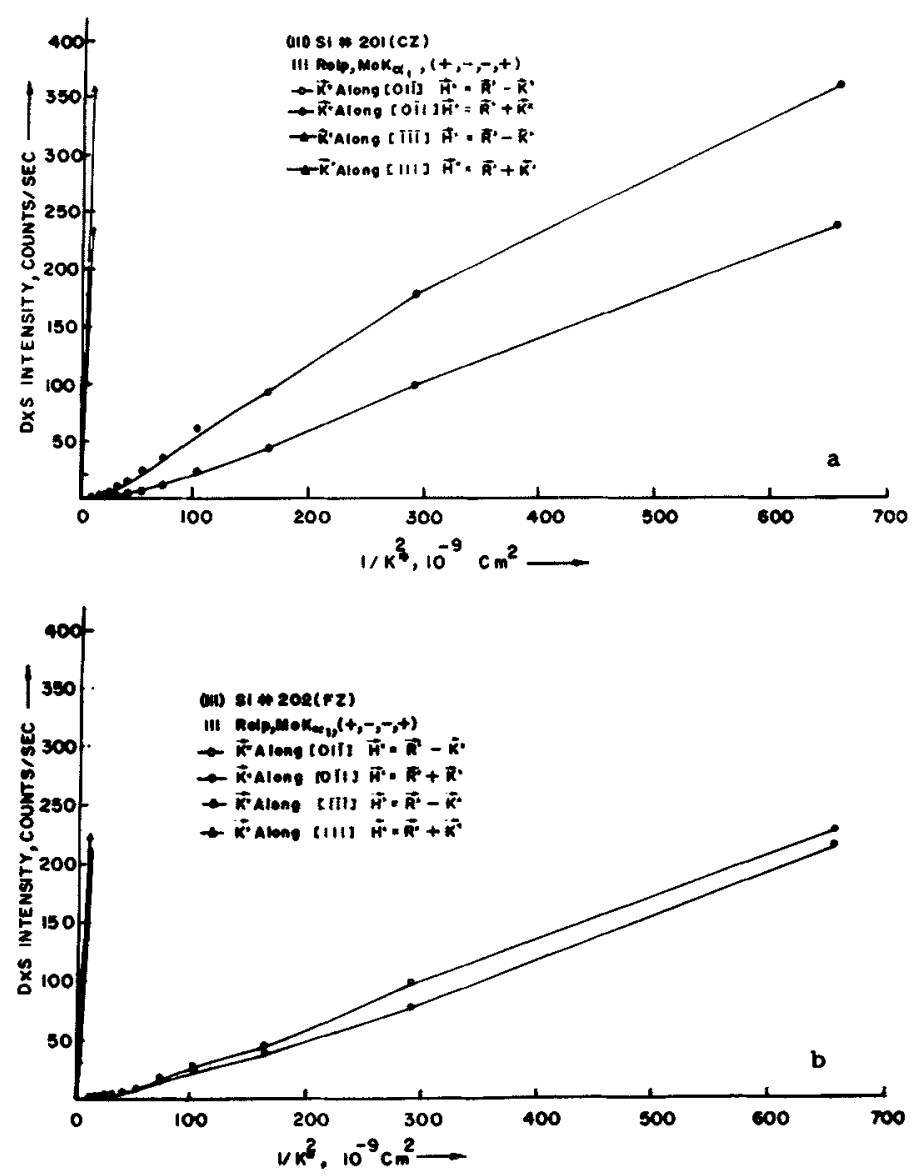

Figure 9. Plots of diffuse $\mathrm{X}$-ray scattering intensity as a function of $\mathrm{K}^{*-2}$ for silicon single crystals grown by (a) the Czochralski method and (b) the float zone method.

of low dimensional devices with GaAs crystals (Paccagnella et al 1988; Wurfl et al 1989). Thin metal deposits like other films produce stress at their interface with the substrate crystals (Segmuller et al 1989). The stress influences properties of the crystals and therefore the quality of the devices fabricated on the same. A variety of metallizations have been developed (Paccagnella et al 1988; Wurfl et al 1989). Recently, biaxial stress introduced in GaAs crystals by the following two types of deposits has been investigated in detail (Lal et al 1989, 1990c): Ge-Ni-WSi ${ }_{2}-\mathrm{Au}$ and $\mathrm{Ge}-\mathrm{Au}-\mathrm{Ni}-\mathrm{WSi}_{2}-\mathrm{Au}$ (thickness: Ge, $20 \mathrm{~nm} ; \mathrm{Au}, 5 \mathrm{~nm} ; \mathrm{Ni}, 10 \mathrm{~nm} ; \mathrm{WSi}_{2}, 100 \mathrm{~nm} ; \mathrm{Au}, 100 \mathrm{~nm}$ ). Semi-insulating (SI) and $n$-type substrate crystals were used. These had their surfaces along (100) planes and their sizes were $5 \times 5 \times 0.1 \mathrm{~mm}^{3}$ or $10 \times 10 \times 0.1 \mathrm{~mm}^{3}$. In this investigation blank wafers without metallizations were also investigated to monitor the changes on metallizations. Besides stress measurements, the perfection of all the specimen crystals was evaluated by diffractometry and topography. This helped in monitoring the degradation in perfection, generation of stress and detection of possible plastic deformation. Also, distribution of stress could be monitored. It may be mentioned 
that special care has to be taken to mount the specimen on the diffractometer. This process by itself should not introduce appreciable stress in the specimen. This is one of the most difficult parts of the whole experiment.

The SI-GaAs blank wafers had low dislocation density as expected of the Bridgman grown crystals. No cellular structure was observed in the topographs. A topograph of a blank wafer has been shown in figure 6 above. Diffraction curves were fairly sharp with half widths of about 15 arcsec. Keeping in view the fact that the monochromators were silicon single crystals producing dispersion broadening, the half width is fairly reasonable. Recently, we have improved the surface preparation of blank wafers and sharper diffraction curves have been obtained.

Deposition of multilayer metallization produced fairly strong bending of the wafers. Biaxial stress has been determined from the experimentally measured value of the radius of curvature $R$ of lattice planes and the thicknesses of the films and the wafers by using the relation

$$
\sigma=\frac{E}{6(1-v)} \frac{t_{s}^{2}}{t_{f}} \frac{1}{R} .
$$

Here $E$ and $v$ are respectively the elastic constant and the Poisson's ratio of the substrate crystal, $t_{s}$ the thickness of the substrate, $t_{f}$ the thickness of the film, and $R$ the radius of curvature of the substrate.

Figure 10 shows a typical set of curvature plots whose slopes give the value of $R$. Curves 1,2 and 3 are, respectively, for a blank wafer, a wafer with $\mathrm{Ge}-\mathrm{Ni}-\mathrm{WSi}_{2}-\mathrm{Au}$ metallization, and a wafer with Ge-Au-Ni-WSi ${ }_{2}-\mathrm{Au}$ metallization. All the substrates

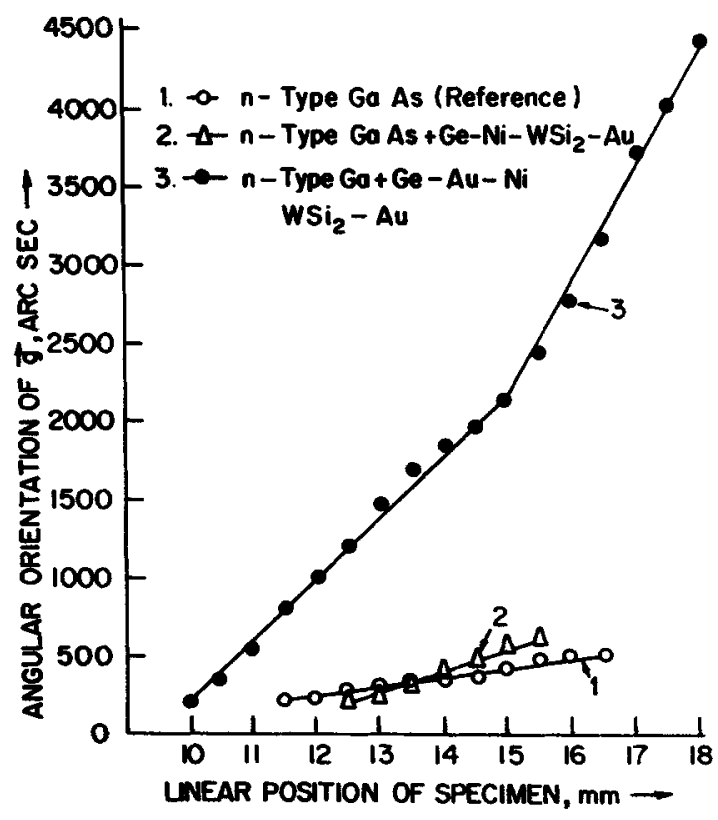

Figure 10. A typical set of curvature plots obtained with $n$-type GaAs crystals. Curves 1.2 and 3 are, respectively, for a blank wafer, a wafer with the four layered deposition (Ge-Ni$\mathrm{WSi}_{2}-\mathrm{Au}$ ) and a wafer with the five layered structure (Ge-Au-Ni-WSi${ }_{2}-\mathrm{Au}$ ). 
were $n$-type (100) GaAs crystals. The slope of curve 1 is the lowest showing the low degree of curvature or bending. The crystal with five layered metallization has the highest value of curvature (curve 3). It may be noted that curve 3 has two straight line segments, which are due to two blocks of deposits. The biaxial stress has been determined as $1 \times 10^{10}$ dyne $\mathrm{cm}^{-2}$ for the crystal with four layered metallization (curve 2); and $4.5 \times 10^{10}$ dyne $\mathrm{cm}^{-2}$ and $2.7 \times 10^{10}$ dyne $\mathrm{cm}^{-2}$ for the two blocks of the five layered metallization (curve 3 ). This data clearly show that the stress produced by the five layered deposit is much higher than that produced by the four layered structure. In the case of SI crystals with five layered metallization $\mathrm{Ge}-\mathrm{Au}-\mathrm{Ni}-\mathrm{WSi}_{2}-\mathrm{Au}$ also, large bending was observed. However, the bending was smaller than that observed with the $n$-type crystals. Values of biaxial stress were in the range $0.6-1.7 \times 10^{10}$ dyne $\mathrm{cm}^{-2}$, much less than the stress values observed with $n$-type crystals having the same metallizations.

The stress induced by metallizations deteriorates the crystalline perfection of the substrate crystals as shown by the broadening of the diffraction curves (Lal et al 1990c). In the case of SI crystals the diffraction curve half width increased to 45-155 arcsec from 15 arcsec of the blank crystal. Similarly, large increase in the diffraction curve half widths was observed in $n$-type crystals with depositions. A part of the broadening is due to finite width of the exploring X-ray beam in the plane of diffraction $(0.2 \mathrm{~mm})$.

Interesting results on non-homogeneity and anisotropy in the distribution of stress could be observed in our experiments. X-ray diffraction topographs revealed that the stress is nonhomogeneously distributed. Band-like structure is clearly seen. A typical topograph with these features is shown in figure 11 . Nearly vertical band-like images

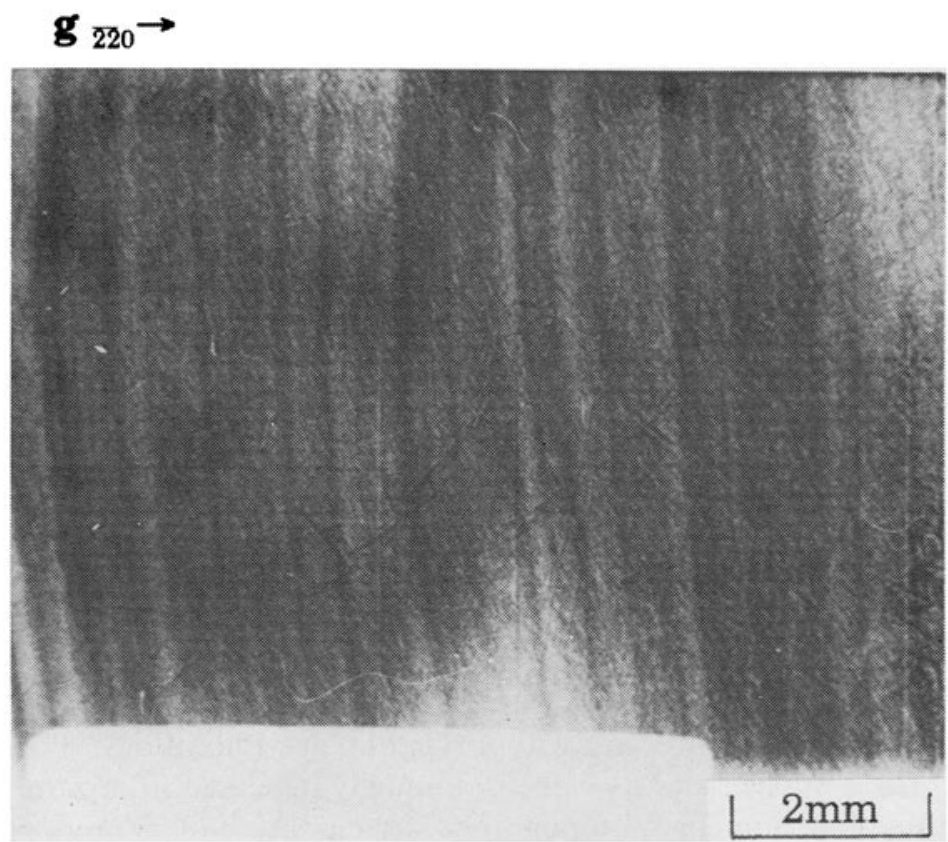

Figure 11. A typical traverse topograph of a SI GaAs with five layered metallization. Mo $K \alpha_{1}$ radiation and ( $\left.\overline{2} \overline{2} 0\right)$ diffracting planes were employed. 
are clearly observed. The bands are perpendicular to the diffraction vector, showing that the strain is also perpendicular to their lengths. Diffraction curves were recorded at different locations of the specimen. It was observed that there is good correlation between the stress levels observed in the topographs and the values of the half widths of the diffraction curves.

Anisotropy in stress distribution was experimentally determined by performing experiments with different sets of diffracting planes. Some of these were parallel to the surface of the specimen crystals and were investigated in the Bragg geometry. However, most of the planes were perpendicular to the surface and were investigated in the Laue geometry. The stress in the lattice planes parallel to the surface does not show band-like distribution in topographs. Also, the value of biaxial stress was different for different sets of lattice planes.

The stress distribution revealed by these experiments is due to the combined effect of the lattice mismatch between the substrates and the deposits, mismatch between thermal coefficients of expansion, and chemical reaction between the deposited layers and the substrates.

\subsection{Characterization of microstructural defects produced in silicon single crystals by externally applied electric fields}

The sensitivity of the multicrystal X-ray diffractometers developed in our laboratory is so high that even small microstructural defects can be directly photographed. This capability has been utilized in a study of real structure of single crystals when electric field was applied (Lal and Thoma 1981, 1985; Lal and Goswami 1987; Lal 1988b). A four crystal X-ray diffractometer was employed (Lal, N P L Report 1985; Lal 1988b, c; Lal and Goswami 1987). It has two plane (111) silicon single crystal monochromators of Bonse-Hart type (Bonse and Hart 1965). The specimen crystal to be investigated is taken in the form of a circular disc. Conducting electrodes (thin aluminium films) are deposited on the two flat surfaces and connected to a source of electrical power. The specimen is placed at the third crystal position of the diffractometer and oriented for diffraction. The fourth crystal is used as an analyser whose main purpose is to monitor any lattice parameter changes which might be induced by the electric field either by itself or through heating. Diffraction curves, topographs and curvature measurements are made before, during and after the application of electric field. Experiments have been performed at a few discrete frequencies but covering a wide range: $0 \mathrm{~Hz}$ (dc field) to a few $\mathrm{GHz}$. At microwave frequencies, however, the electrodes have to be prepared differently. A stripline is deposited on one side of the crystal while the other side is covered entirely with an aluminium film (Lal and Thoma 1981). The electric power loss density ( $p$ ) defined as the electrical power lost in a unit volume of the crystal has been varied in a range covering several orders of magnitude. Some typical results are briefly described in the following.

Diffraction curves of specimen silicon single crystal recorded before the application of the electric field were very sharp with a typical half width of only 3 arcsec. When an electric field is applied and its value is gradually increased from zero, no change is observed in diffraction curves, topographs and curvature plots up to small power densities of $\leqslant 0.01 \mathrm{~W} \mathrm{~mm}^{-3}$. However, remarkable changes are observed in the high resolution traverse topographs above threshold value of $p$. For silicon crystals, this 


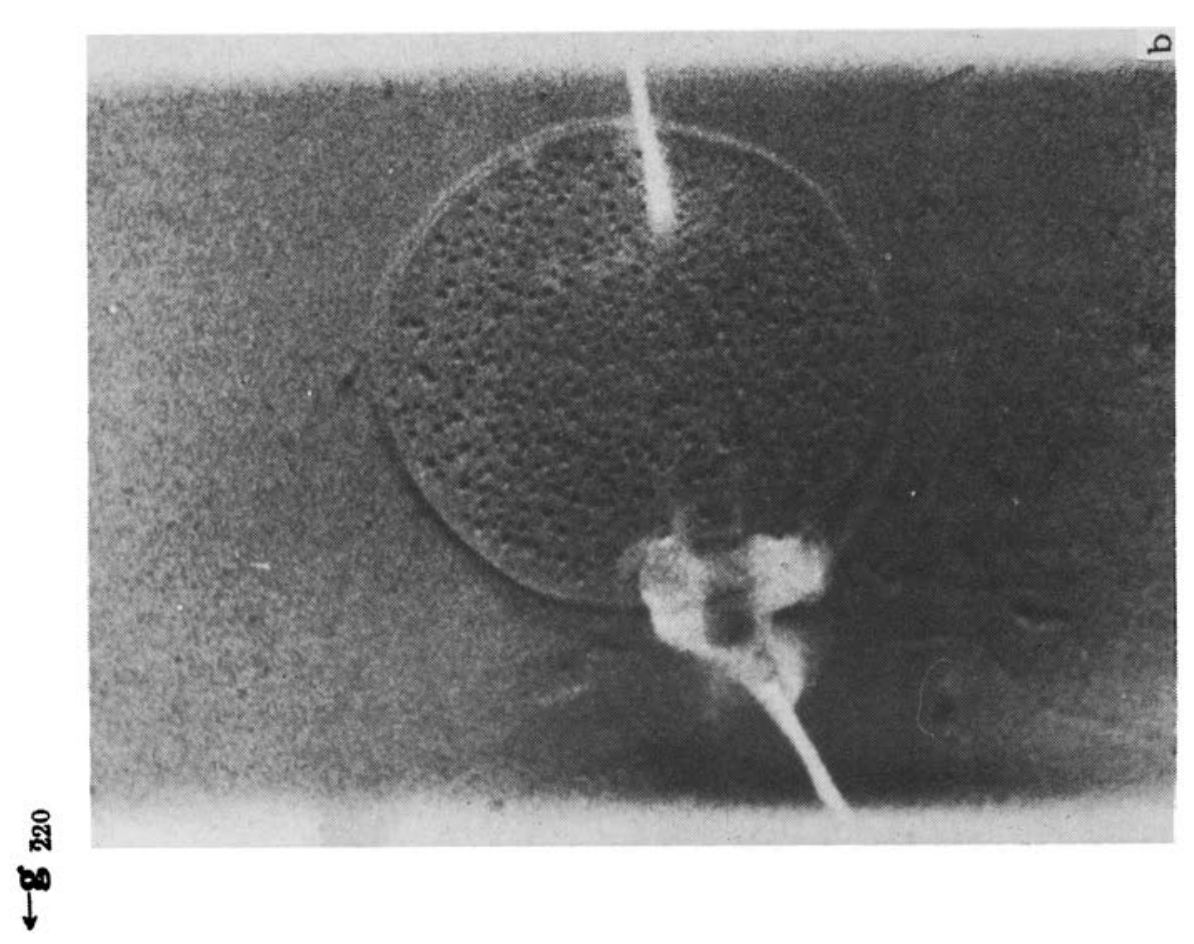

E

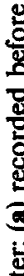

ष्ष

รัํำ

象

究

安

焉

敋

흥

* II

5

롱 끌

는

若

존

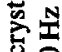

苍营

$\pi$

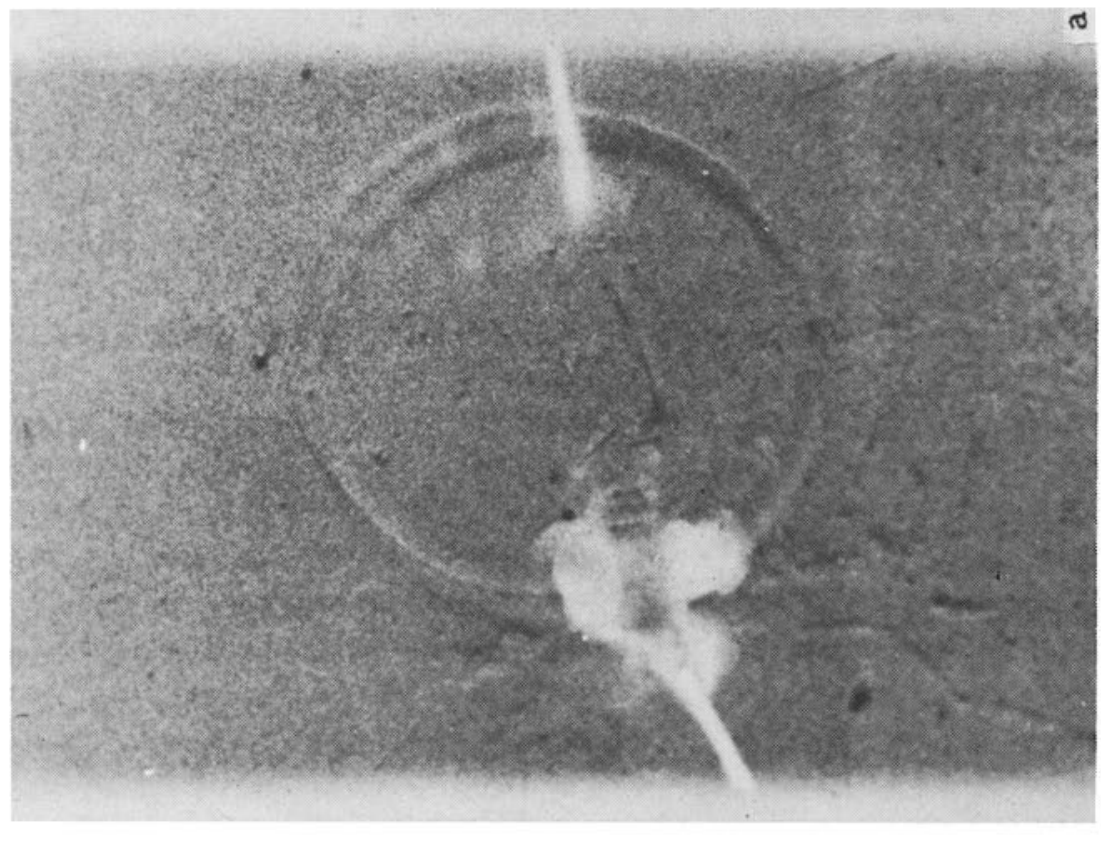

E

\%

零

욤

过

용

岁怘

主

5 웜

. 둔

.

荧

画

존

$x$ 긍

ธี

吾

些。

ผึ

슬 
threshold value of $p$ has been determined as $\sim 0.01 \mathrm{~W} \mathrm{~mm}^{-3}$. Figures 12 (a) and (b) show a set of two high resolution traverse topographs recorded before and during the application of an electric field (corresponding to $p=0.01 \mathrm{~W} \mathrm{~mm}^{-3}$ ), respectively. Before the field is applied the topograph shows only a few details, like the contours of the circular electrodes and the shadows of the connecting wires. No dislocation or any other major defect is visible, except one or two surface features (figure 12a). However, in the second topograph recorded while the crystal was under an electric field, a lot of dot-like features are observed in the electrode region. These features have strong black and white contrast which can be analysed by changing the sense of the diffraction vector (Lal and Goswami 1987). This analysis shows that the regions under dots are pushing out the surrounding matrix and the stress makes these visible. The diameters of these images are in the range $100-160 \mu \mathrm{m}$. The sizes of the defects

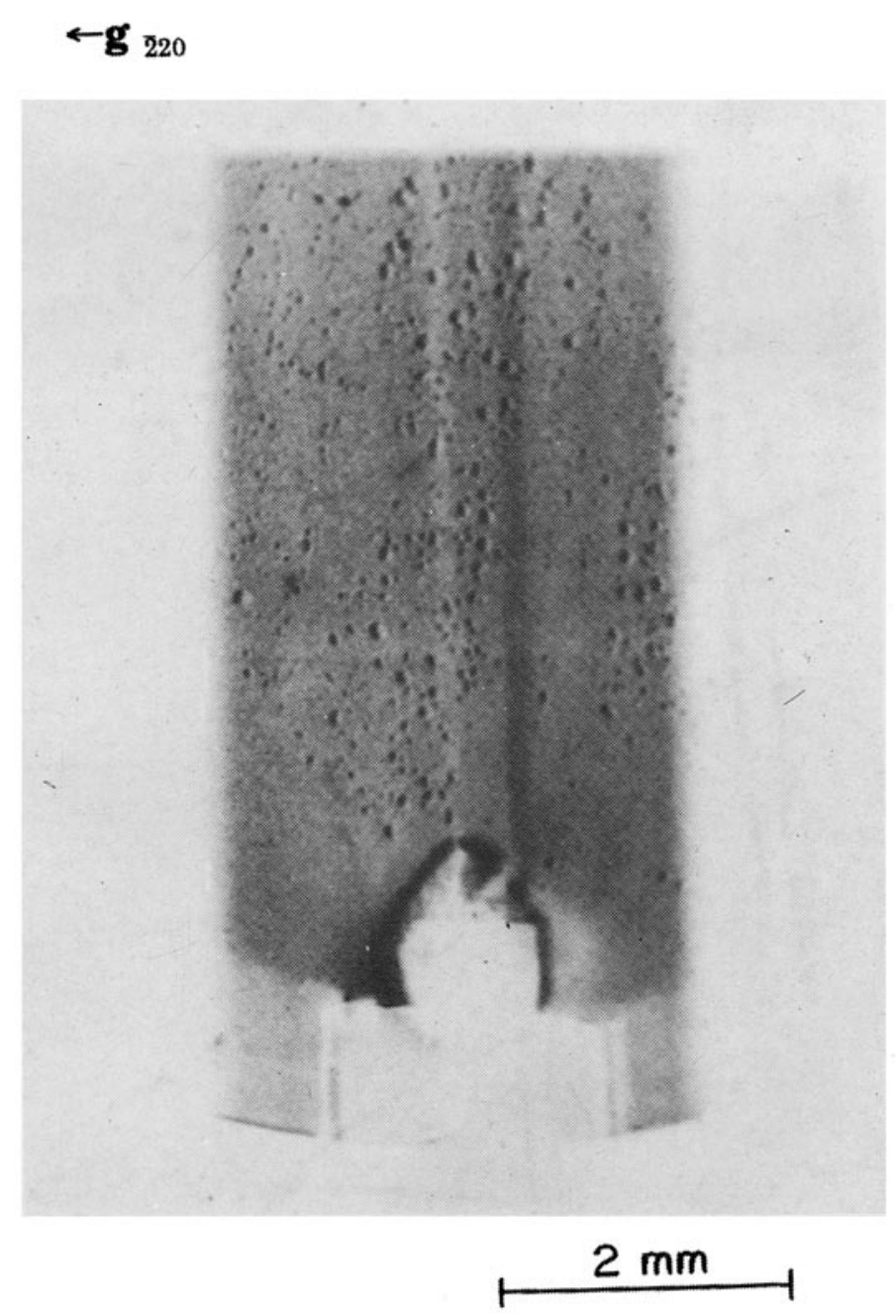

Figure 13. A high resolution X-ray diffraction (traverse) topograph of a silicon crystal recorded while an $2.45 \mathrm{GHz}$ electric field was applied to the crystal. 
responsible for these images are expected to be very small in comparison to the observed diameters. This is due to the high sensitivity of the technique employed for their observation. At this value of $p$ there was no significant change in the shapes of the diffraction curves. Also, there was no significant rise in the average temperature of the specimen (particularly in the volume between electrodes) as revealed by the absence of any lattice parameter increase monitored with the help of the analyser crystal of the diffractometer. The analyser has been demonstrated to pick up a few degrees rise in temperature (Lal and Thoma 1983). It may be mentioned that microwave fields of $2.45 \mathrm{GHz}$ frequency also produce dot-shaped images which exhibit similar black and white contrast. Figure 13 shows a typical topograph which shows this result.

The microstructure observed on application of the electric field with $p$ values above the threshold value varies from specimen to specimen. The sizes and shapes of the images depend upon the specimen, particularly its region under the electrodes. Figure 14 shows a traverse topograph of a silicon crystal recorded when it was subjected to dc electric field $\left(p \sim 0.027 \mathrm{~W} \mathrm{~mm}^{-3}\right)$. This topograph covers a large circular region around the electrodes. Electric field induced microstructure is clearly seen in the electrode region. However, the density of the defects is much lower in this case in comparison to figure 12b. An interesting feature in this topograph is the image of a handwritten $A$, around which a small circle has been drawn for convenience

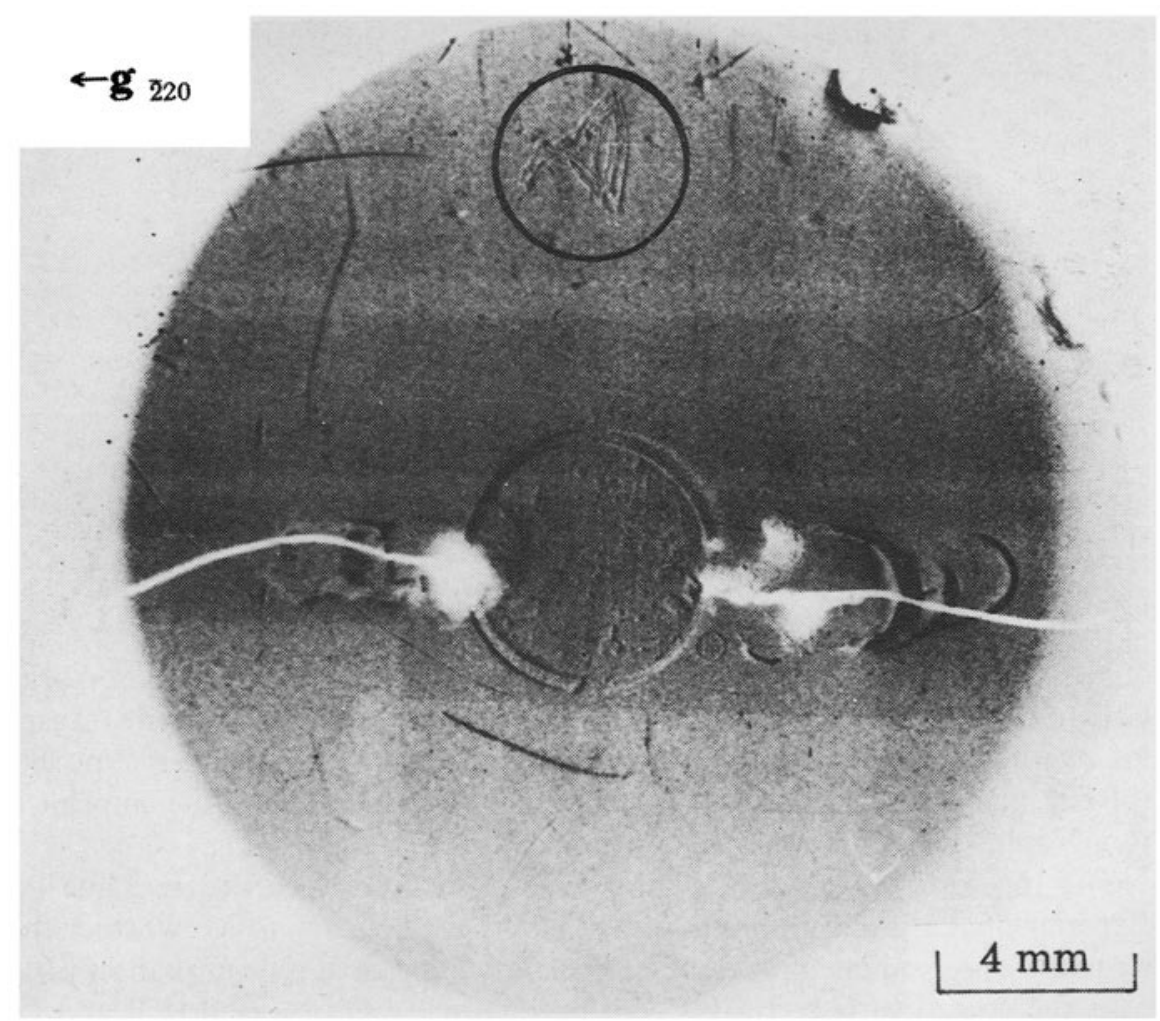

Figure 14. A high resolution traverse topograph of a silicon single crystal, recorded while it was subject to an electric field $(0 \mathrm{~Hz})$ corresponding to $p=0.027 \mathrm{~W} \mathrm{~mm}^{-3}$. 


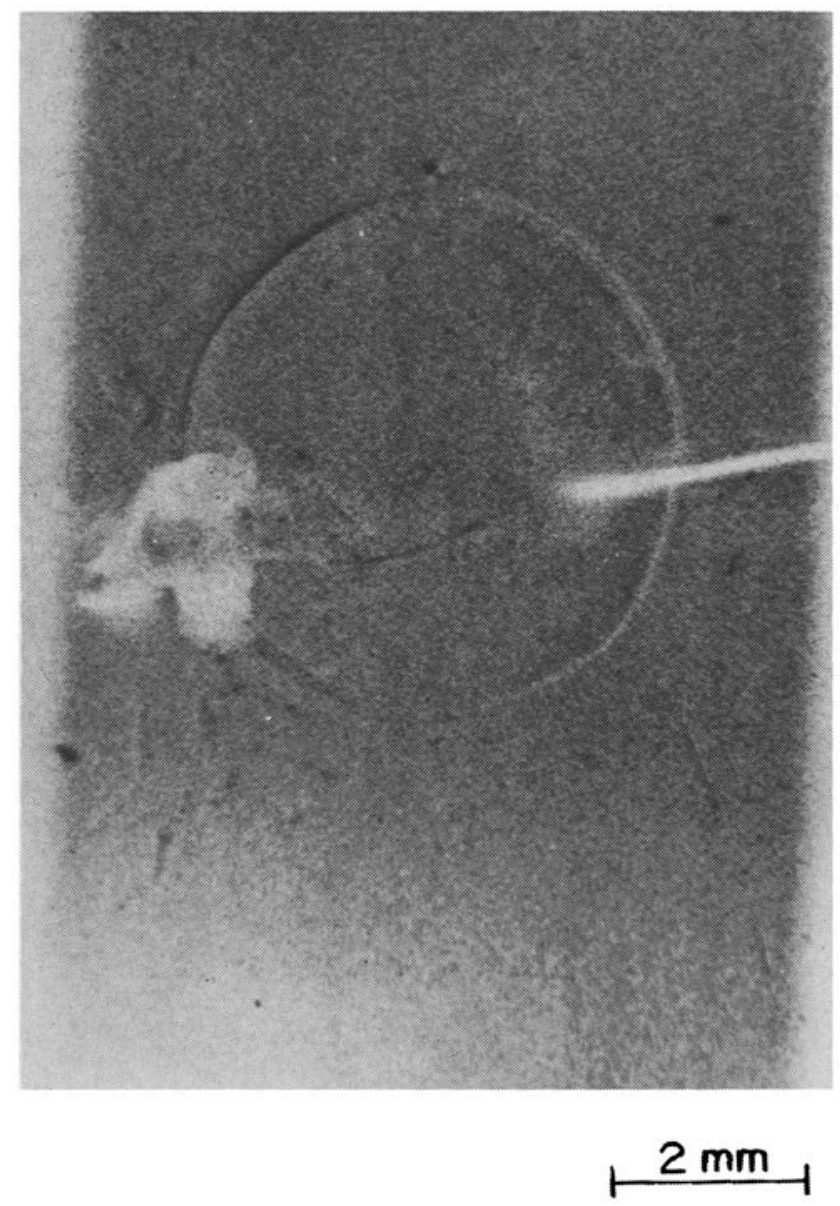

Figure 15. A high resolution traverse topograph of a silicon single crystal recorded 57 days after the field was switched off. The topograph recorded under the field has been shown in figure 12(b).

of observation. Before the deposition of electrodes the specimens were marked A, B, $\mathrm{C}, \mathrm{D}$ to distinguish one from the other. A felt pen generally used to write transparencies for overhead projection was employed. These writings were later removed with a solvent like acetone. However, the crystal lattice preserves the imprint and the topographic technique is sensitive enough to reveal the writing.

The topographs shown in figures 12 and 13 were recorded in Laue geometry. Experiments have also been performed in Bragg geometry to see whether the microstructure observed on application of electric field originates from shallow dislocations near the crystal surface. In this case no contrast was observed (Lal and Goswami 1987), showing that the disturbance in the real structure is in the volume of the specimen crystals. 


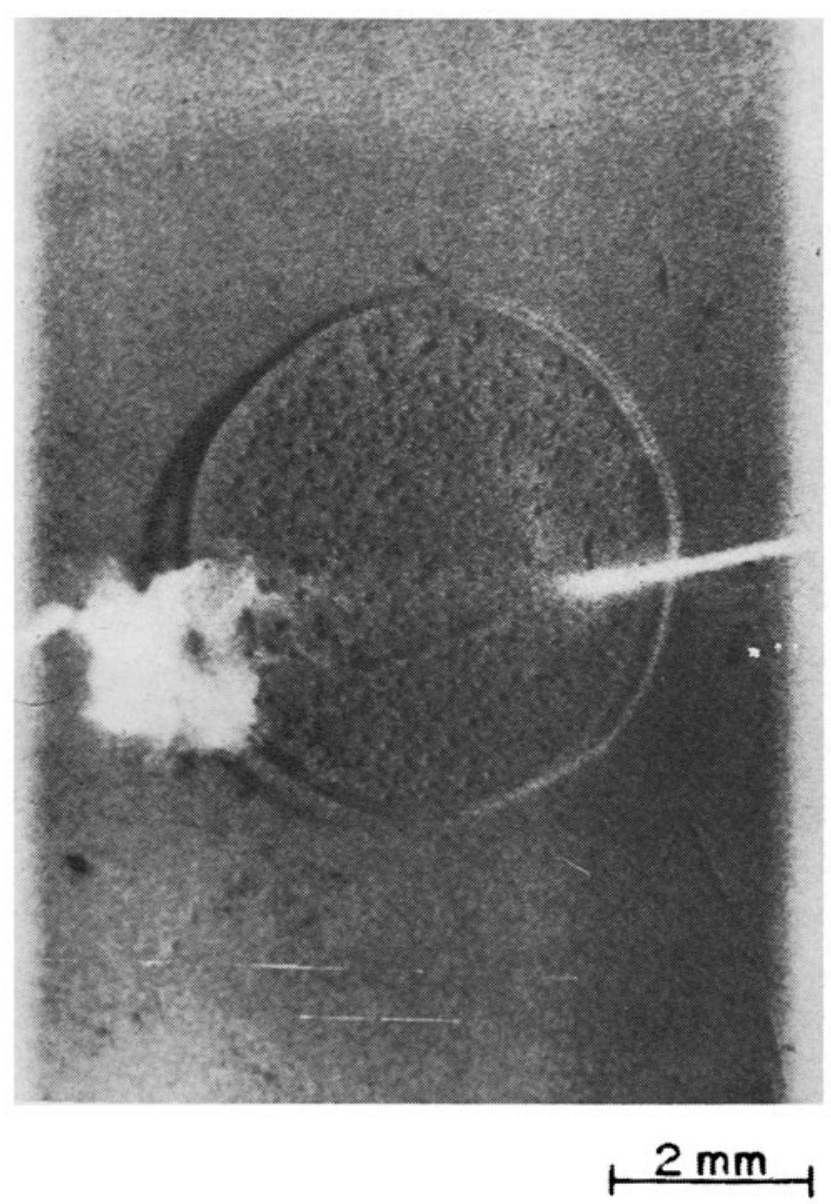

Figure 16. A high resolution traverse topograph of the silicon crystals of figures 12 and 15. This was recorded while the crystal was subjected to electric field a second time after the microstructure observed on the first application of electric field bad faded out.

The microstructure induced by electric field has been found to be quite stable, even after the field is removed. After eight to nine weeks storage of the specimen at room temperature the images completely fade out in topographs as shown in figure 15 . On a second application of the electric field the microstructure identical to that observed on first application of electric field reappears. Figure 16 shows a topograph recorded on second application of the field.

Results of detailed studies on a number of crystals suggest that the images observed on application of electric field to the crystal are associated with microdefects or residual defects such as point defect clusters in dislocation-free crystals. The defects lead to a nonhomogeneous distribution of electric current in the specimen which predominantly flows along 'preferred paths' or 'filaments'. The strain around these 
'filaments' is imaged in the topographs. This work gave the first direct observation of images of filaments.

A very interesting result of the experiments with crystals under high electric field was the remarkable enhancement in intensity of diffracted X-rays when the specimen was subjected to the external field (Lal and Thoma 1985). The enhancements could be by a factor of three or more. In exceptional cases, the increase at microwave frequencies was by a factor of ten (Lal and Thoma 1985; Lal 1988b). Recently, large enhancements have also been observed with dc fields at high power densities (Lal and Goswami, to be published). Attempts are being made to understand changes in diffracted intensities in terms of changes in atomic scattering factors (Bhasin et al 1988).

Experiments have also been performed with crystals of different band gaps like lithium fluoride and cadmium sulphide. All of these crystals exhibit different types of microstructural changes (Lal and Thoma 1981). A remarkable feature is the observation of a one-to-one correlation of fluctuations in electric currents in the crystal and those in the diffracted X-ray intensities (Lal and Thoma 1981; Lal 1988b).

This is an area of considerable excitement and a variety of experiments are being performed to throw light on fundamental aspects connected with this problem.

\section{Direct observation of dynamical features of $\mathrm{X}$-ray diffraction from thin diamond crystals of varying degrees of perfection}

As mentioned earlier, dynamical features of X-ray diffraction are expected only when the crystals are 'thick', defined by $\mu t \geqslant 10$, and nearly perfect. We have developed a new technique which has enabled us to directly observe the following two notable dynamical features with crystals of varying degrees of perfection (Lal et al 1992, 1993): (i) the forward diffracted X-ray beam; and (ii) anomalous transmission of X-rays. Main results of these investigations are briefly described here.

Figure 17 shows a schematic line diagram of the new technique developed for direct observation of forward diffracted beam in 'thin' crystals. The exploring beam is a highly monochromated and collimated $\mathrm{K} \alpha_{1}$ (Mo $\mathrm{K} \alpha_{1}$ in the present study) beam

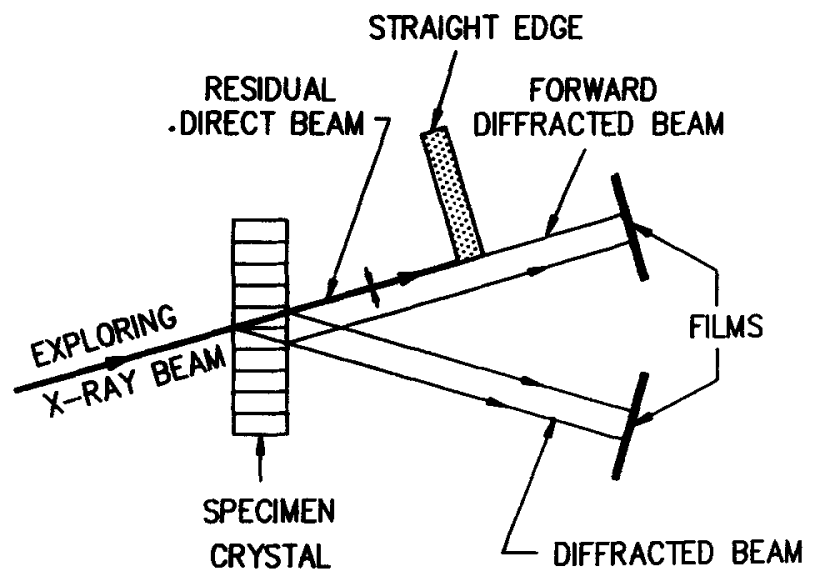

Figure 17. A schematic line diagram showing the basic principle of the new technique developed for direct observation of forward diffracted X-ray beam with thin crystals. 


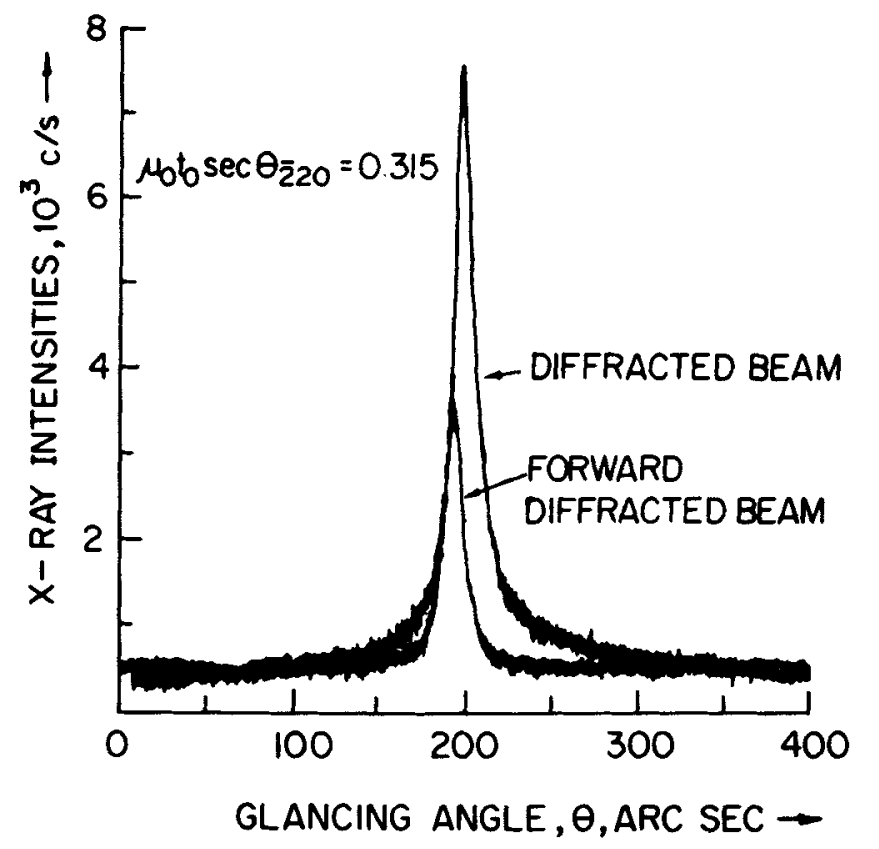

Figure 18. A typical set of two diffraction curves of a (111) natural diamond crystal recorded when the straight edge was introduced to block the residual direct beam. The outer curve is for the diffracted beam and the inner curve is for the forward diffracted beam.

as obtained in the four- and five-crystal diffractometers. The width of this beam in the plane of diffraction is small $(\sim 0.2 \mathrm{~mm})$. The specimen thicknesses corresponded to $\mu t$ values of $\sim 0.3$ or less, going down to 0.1 . These crystals were oriented for diffraction in the Laue geometry. In the forward beam direction, two beams are observed when the condition for diffraction is satisfied. One of these beams is the usual residual direct beam, while the other beam is the forward diffracted beam as shown in figure 17 . The residual direct beam can be blocked by a carefully prepared straight edge. In this process a small fraction of the forward diffracted beam which overlaps the residual direct beam is also blocked. Nevertheless, with this method bulk of the forward diffracted beam can be directly observed.

Figure 18 shows a set of two diffraction curves of a diamond crystal recorded with (220) diffracting planes. The value of $\mu t \sec \theta$ is 0.315 , which is much less than one and, therefore, the crystal can be treated as 'thin'. The outer curve is for the normal diffracted beam while the inner curve is for the forward diffracted beam. The diffraction curves are very sharp showing the high degree of perfection of this specimen. Theoretically at this value of $\mu t \sec \theta$ a dip rather than a peak is expected for the forward diffracted X-ray beam (Lal et al 1992). Figure 19 shows photographs of the exploring beam (a), the diffracted beam (b) and the residual beam + forward diffracted beam (c). The width of the exploring beam is about one fourth of that of the forward diffracted beam. Therefore, the forward diffracted beam can be conveniently observed.

For investigation of the anomalous transmission of X-rays through diamond crystals the total transmitted intensity was measured around the Bragg angle. The straight edge was not employed and the intensity of the two beams in the forward direction 

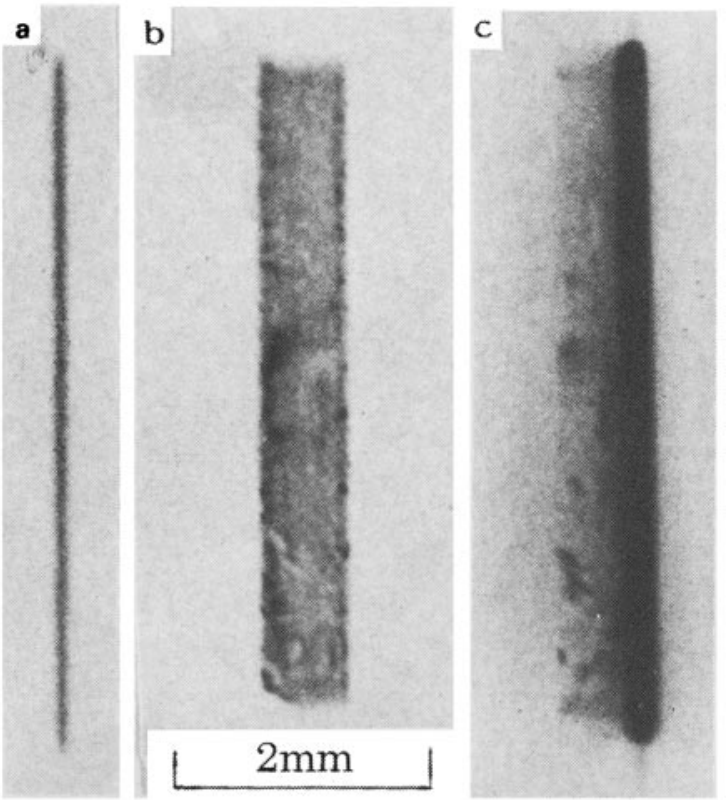

Figure 19. A typical set of photographs of the following $X$-ray beams recorded with a thin (111) natural diamond crystal specimen: (a) the exploring beam; (b) the diffracted beam; and (c) the forward diffracted beam and the residual direct beam.

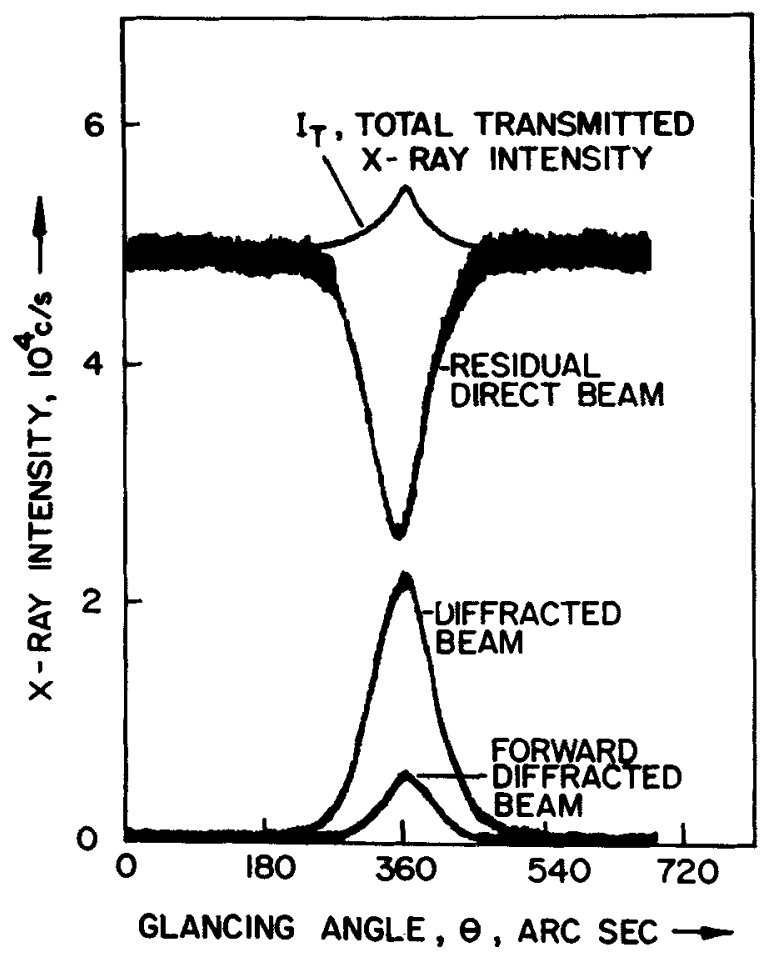

Figure 20. A set of curves of a 'thin' (111) natural diamond crystal. The uppermost curve is for the total transmitted intensity through the crystal which shows a peak at $\theta_{\mathbf{B}}$. 
was measured together. Figure 20 shows a plot of the total transmitted intensity through the specimen. The other curves are also shown in the figure. It is observed that the total transmitted intensity through the specimen shows a peak at the crystal orientation for the diffraction maximum. This clearly shows that X-ray transmission is enhanced at the diffraction peak (Lal et al 1993), or Borrmann effect is observable. One has to keep in view that these were thin crystals.

Recently, we have succeeded in observing forward diffracted beam as well as Borrmann effect with diamond crystals which were not only 'thin' $(\mu t \ll 1)$ but also rather imperfect (Lal et al 1993). These remarkable observations have been possible due to the high quality of the X-ray beam employed in these experiments.

\section{Concluding remarks}

In this article an attempt has been made to present an overview on characterization of real structure of single crystals of technologically important materials by using high resolution $\mathrm{X}$-ray diffraction techniques and multicrystal X-ray diffractometers developed in our laboratory. A review of this length cannot be exhaustive in terms of theory, experimental techniques and illustrative examples. This area offers ever increasing challenges to uncover atomic order in crystals and its relationship with crystal properties and external influences.

\section{Acknowledgements}

Partial financial assistance received from the Department of Science and Technology, New Delhi (under Indo-US projects) is acknowledged with pleasure. Part of the work reported here has been carried out under an Indo-FRG collaborative project. The author is thankful to Dr S N N Goswami for many fruitful discussions.

\section{References}

Batterman B W and Cole H 1964 Rev. Mod. Phys. 36681

Bhasin V S, Kothari L S, Lal K and Srivastava M P 1988 Phys. Letts. A133 438

Bonse U and Hart M 1965 Appl. Phys. Lett. 7238

Borrmann G 1950 Z. Phys. 127 S297

Compton A H and Allison S K 1935 X-rays in theory and experiment (New York: Van Nostrand)

Darwin C G 1914 Philos. Mag. 27 315, 675

Dederichs P H 1973 J. Phys. F3 471

Ehrhart P, Trinkaus H and Larson B C 1982 Phys. Rev. B25 834

Ewald P P 1916 Ann. Phys. 49 1, 117

Ewald P P 1917 Ann. Phys. 54519

Iida A and Kohra K 1979 Phys. Status Solidi a51 533

James R W 1950 The optical principles of the diffraction of $X$-rays (London: Bell $G$ and Sons)

James R W 1963 Solid State Phys. 1555

Kato N 1974 in X-ray diffraction (ed.) L Azaroff (New York: McGraw Hill)

Kato M 1980 Acta Crystallogr. A36 763

Krivoglaz M A 1969 Theory of X-ray and thermal neutron scattering by real crystals (New York: Plenum)

Lal K (ed.) 1982 Synthesis, crystal growth and characterization (Amsterdam: North-Holland) pp 215 and 287

Lal K 1985 NPL Technical Report NPL-85-B4-0051 
Lal K 1988a in 40 Years of research - A CSIR overview series II (New Delhi: CSIR) p. 1

Lal K 1988b Rigaku J. 511

Lal K 1988c Cryst. Prop. Prep. 16143

Lal K 1989 Prog. Cryst. Growth Charact. 18227

Lal K 1991a Proc. Indian Nat. Sci. Acad.

Lal K 1991b Key Engg. Mater. 58205

Lal K and Singh B P 1977 Solid State Commun. 2271

Lal K and Singh B P 1979 Indian J. Phys. A53 72

Lal K and Singh B P 1980 Acta Crystallogr. A36 178

Lal K and Thoma P 1981 Solid State Commun. 2271

Lal K and Thoma P 1983 Phys. Status Solidi A80 491

Lal K and Thoma P 1985 Solid State Common. 53107

Lal K and Verma A R 1984 Bull. Mater. Sci. 6129

Lal K and Goswami S N N 1987 Mater. Sci. Engg. 85147

Lal K and Goswami S N N 1988 Rev. Sci. Instrum. 591409

Lal $\mathrm{K}$ and Goswami $\mathbf{S} \mathbf{N} \mathbf{N}$ (to be published)

Lal K and Bhagavannarayana G 1989 J. Appl. Crystallogr. 22209

Lal K and Bhagavannarayana G 1991 J. Appl. Phys. 698092

Lal K, Bhagavannarayana G, Kumar V and Halder S K 1990 Meas. Sci. Technol. 1793

Lal K, Singh B P and Verma A R 1979 Acta Crystallogr. A35 286

Lal K, Goswami S N N, Wurfl J and Hartnagel H L 1989 J. Semiconduct. Dev. 1124

Lal K, Goswami S N N and Verma A R 1990a Pramana - J. Phys. $3 \dot{4} 507$

Lal K, Goswami S N N and Verma A R 1990b Solid State Commun. 75881

Lal K, Goswami S N N, Wurf J and Hartnagel H L 1990c J. Appl. Phys. 674105

Lal K, Goswami S N N and Verma A R 1992 Solid State Commun. 81461

Lal K, Goswami S N N and Verma A R 1993 XVI Congress Int. Union Crystallography, Abs. No. PS 14.03.07

Laudise R A 1975 in Crystal growth and characterization (eds) R Ueda and J B Mullin (Amsterdam: North Holland) p. 255

von Laue M 1960 Rontegenstrahlinterferenzen (Frankfurt: Akad. Verlagsgesellschaft)

National Acad. Sci. and National Acad. Engg. Comm. Materials Advisory Board - MAB Report 229-M 1967 Washington D.C.

Paccagnella A, Canali C, Donzelli G, Zanoni E and Wang C L 1988 Electron. Lett. 24788

Patel J R 1973 J. Appl. Phys. 443903

Patel J R 1975 J. Appl. Crystallogr. 8186

Pinsker Z G 1978 Dynamical scattering of $X$-rays in crystals (Berlin: Springer Verlag)

Schneider J R, Bouchard R, Graf H A and Nagasawa H 1992 Acta Crystallogr. A48 804

Segmuller A, Noyan I C and Speriosu V 1989 Prog. Cryst. Growth Charact. 1821

Tanner B K 1976 X-ray diffraction topography (Oxford: Pergamon Press)

Wooster W A 1962 Diffuse $X$-ray reflections from crystals (Oxford: Clarendon Press)

Wurfl J, Nassibian A G, Hartnagel H L, Langfeld R and Mauer C 1989 Int. J. Electron. 66213

Zachariasen W H 1945 The theory of $X$-ray diffraction in crystals (New York: John Wiley and London: Chapman and Hall)

Zaumseil F, Winter U, Cembali F, Servidori M and Sourek Z 1987 Phys. Status Solidi A100 95 